\title{
miR-451a suppression of IL-6R can inhibit proliferation and increase apoptosis through the JAK2/STAT3 pathway in multiple myeloma
}

\author{
LING ZHONG $^{1,2^{*}}$, ZHUYU XU $^{3 *}$, XIN JIN $^{1 *}$, YUAN HE $^{1}$, JIANBO ZHANG $^{1}$, TAO JIANG ${ }^{4}$ and JIAO CHEN ${ }^{4}$ \\ ${ }^{1}$ Department of Clinical Laboratory, Sichuan Academy of Medical Science \& Sichuan Provincial People's Hospital, \\ School of Medicine, University of Electronic Science and Technology of China, Chengdu, Sichuan 610072; \\ ${ }^{2}$ Chengdu Institute of Biology, Chinese Academy of Sciences, Chengdu, Sichuan 610041; ${ }^{3}$ Department of Pharmacy, \\ Sichuan Academy of Medical Science \& Sichuan Provincial People's Hospital, Personalized Drug Therapy Key \\ Laboratory of Sichuan Province, School of Medicine, University of Electronic Science and Technology of China, \\ Chengdu, Sichuan 610072; ${ }^{4}$ Department of Hematology, Sichuan Academy of Medical Sciences \\ \& Sichuan Provincial People's Hospital, Chengdu, Sichuan 610072, P.R. China
}

Received December 6, 2019; Accepted August 25, 2020

DOI: $10.3892 / \mathrm{ol} .2020 .12202$

\begin{abstract}
The IL-6R/JAK2/STAT3 pathway mediated by interleukin-6 (IL-6) plays an important role in the occurrence and development of multiple myeloma (MM), which is associated with decreased microRNA-451a. However, the biological function of microRNA-451a in MM remains unclear. The bone marrow (BM) of patients with MM was sampled, and the plasma cells were enriched. BM miR-451a, IL-6 and IL-6R levels and Ki-67 expression intensity were evaluated using reverse transcription-quantitative PCR, ELISA and flow cytometry, respectively. U266 cell proliferation, viability and apoptosis were measured using BrdU, CCK-8 and Annexin V/propidium iodide assays, respectively. Total and phospo-(p-) JAK2 and p-STAT3 levels were measured by western blotting. Dual-luciferase reporter assays were performed to validate the predicted target binding sites. miR-451a expression was low in patients with MM and was associated with the Revised International Staging System (R-ISS) stage. IL-6 concentrations were significantly higher in patients with MM than in normal controls and were inversely associated with miR-451a levels $(r=-0.96, P<0.0001)$. IL-6R levels were positively correlated with the R-ISS stage. miR-451a was downregulated, and
\end{abstract}

Correspondence to: Professor Tao Jiang or Professor Jiao Chen, Department of Hematology, Sichuan Academy of Medical Sciences \& Sichuan Provincial People's Hospital, 32 West Section 2, 1 Ring Road, Chengdu, Sichuan 610072, P.R. China

E-mail: suyeku@126.com

E-mail: chenjiaoxy@med.uestc.edu.cn

*Contributed equally

Key words: microRNA-451a, interleukin-6R, JAK2/STAT3, multiple myeloma
IL-6R was upregulated in myeloma cell lines. Treatment with an miR-451a mimic inhibited viability and induced apoptosis in U266 cells. p-JAK2 and p-STAT3 levels were significantly lower in mimic-treated U266 cells than in control cells. Thus, miR-451a was shown to regulate myeloma cell proliferation and apoptosis via the IL-6R/JAK2/STAT3 pathway and may be used to predict patient prognosis.

\section{Introduction}

Multiple myeloma (MM) is one of the most common hematologic malignancies and accounts for $\sim 1 \%$ of all cancer cases (1). Although MM has been studied for 150 years, it still remains an incurable disease (2). In recent years, immunomodulatory agents such as lenalidomide and proteasome inhibitors (PIs), including bortezomib, have substantially improved the survival of patients with MM. However, patients with MM may acquire drug resistance and thus exhibit recurrence and progression (3). Therefore, investigating the pathogenic mechanisms underlying the development and progression of MM is vital, particularly in regard to proliferation and apoptosis, to identify novel treatment strategies.

Interleukin-6 (IL-6) is a pivotal growth factor that mediates the proliferation of MM via autocrine mechanisms when released from MM cells or via paracrine mechanisms when released from stromal cells (4). IL-6 exerts its biological activities by binding to its receptor (IL-6R). The levels of both IL-6 and IL-6R are upregulated in patients with MM (5). IL-6R leads to activation of the JAK-STAT signaling pathway to regulate the proliferation and apoptosis of myeloma cells (6). However, the mechanism by which IL-6R is upregulated in MM is still unknown.

MicroRNAs (miRNAs/miRs) bind to the 3'-untranslated regions (3'-UTRs) of specific target mRNAs, thereby inhibiting the expression of the target genes post-transcriptionally. miR-451a was discovered in 2005 and is located on chromosome $17 \mathrm{q} 11.2$ (7). miR-451a acts as a tumor suppressor and 
is associated with several types of cancer, including lung cancer (8), hepatocellular carcinoma (9), glioma (10) and osteosarcoma (11), and inhibits the proliferation, migration and invasion of tumors (12). miR-451a targets IL-6R in several types of solid tumors and inhibits tumor proliferation, migration and angiogenesis through the IL-6R signaling pathway (13). However, whether miR-451a also inhibits tumor growth in MM and targets IL-6R remains unknown. Additionally, the clinical significance of miR-451a in MM has not been established. The aim of the present study was to address these gaps in our knowledge.

\section{Materials and methods}

Ethics statement. The present study was approved by the Institutional Review Board of Sichuan Provincial Peoples' Hospital of China. Written informed consent was obtained from each eligible patient, and the study was performed in accordance with the guidelines stated in the Declaration of Helsinki.

Study subjects. The diagnostic criteria for active MM were based on the International myeloma working group (IMWG) guidelines (14). Because the study did not involve any interventions, a sample meeting the diagnostic criteria was considered acceptable. The sub-typing criteria were based on subtypes of abnormal proliferative immunoglobulin (14). The staging criteria were based on the Revised International Staging System (R-ISS) (14). In total, 10 unrelated control subjects from among donors were recruited. Disease responses were assessed according to the IMWG guidelines (15). A total of 66 patients with MM (37 men and 29 women; age range, 38-88 years; median age, 63.0 years) and 10 healthy controls (six men and four women; age range, 31-83 years; median age, 59 years) were enrolled in the present study between January 2017 and December 2018.

Sampling. Bone marrow (BM) aspirates were collected from normal controls and patients after obtaining informed consent and were subjected to red blood cell lysis using Versalyse Lysing Solution (cat. no. A09777; Beckman Coulter, Inc.) and mononuclear cell isolation. Mononuclear cells were isolated using a Ficoll gradient (density $1.077 \mathrm{~g} / \mathrm{ml}$ ) with Lymphoprep (cat. no. 07801; STEMCELL Technologies, https://www.stemcell.com/products/lymphoprep.html). Subsequently, plasma cells (PCs) were incubated and separated by positive selection using CD138-coated magnetic beads (Miltenyi Biotec, $\mathrm{GmbH}$ ) according to the manufacturer's protocol. RNA from PCs was extracted to analyze miR-451a levels, and total protein was extracted to assess IL-6R. The remaining BM was used for multiparameter flow cytometry (MFC). The patients were sampled at initial diagnosis and at the indicated time points during the follow-up.

Cell culture. U266 and INA-6 cells were purchased from the National Infrastructure of Cell Line resource and kindly provided by Dr. Gramatzki (Laboratory of Hematology, GIGA-I3, University of Liège, Belgium), respectively, and were maintained in RPMI 1640 medium with GlutaMAX ${ }^{\mathrm{TM}}$ supplemented with $10 \%$ fetal calf serum and $1 \%$ penicillin/streptomycin. All these reagents were purchased from Thermo Fisher Scientific, Inc.

miR-451a transfection. For miR-451a mimic transfection, an miR-451a mimic (5'-AAACCGUUACCAUUACUGAGUU-3'; $50 \mathrm{nM}$; Guangzhou RiboBio Co., Ltd.) and the corresponding negative control (50 nM; Guangzhou RiboBio Co., Ltd.) were separately transfected into 293T cells (purchased from China Center for Type Culture Collection) in the absence of any other treatments. Transfection was performed using Lipofectamine 3000 (Thermo Fisher Scientific, Inc.). miR-451a expression was measured via reverse transcription-quantitative (RT-q) PCR analysis to confirm successful transfection, and the subsequent effect was determined only in cells expressing miR-451a $48 \mathrm{~h}$ post-transfection.

$R T-q P C R$. Total RNA was isolated from the indicated cells using TRIzol ${ }^{\circledR}$ (Invitrogen; Thermo Fisher Scientific, Inc.) according to the manufacturer's protocol. Then, miR-451a was reverse transcribed with a Bulge-Loop ${ }^{\text {TM }}$ miRNA RT-qPCR Starter kit (Guangzhou RiboBio Co., Ltd.). The reaction conditions were as follows: $42^{\circ} \mathrm{C}$ for $60 \mathrm{~min}$ and $70^{\circ} \mathrm{C}$ for $10 \mathrm{~min}$. Relative quantification was performed via qPCR using the SYBR Green PCR kit (Guangzhou RiboBio Co., Ltd.). The following thermocycling conditions were used: Initial denaturation at $95^{\circ} \mathrm{C}$ for $10 \mathrm{~min}$, followed by 40 cycles at $95^{\circ} \mathrm{C}$ for $2 \mathrm{sec}, 60^{\circ} \mathrm{C}$ for $20 \mathrm{sec}$ and $70^{\circ} \mathrm{C}$ for $10 \mathrm{sec}$. The following primer sequences were used: miR-451a forward, 5'-ACCGTT ACCATTACT-3' and reverse, 5'-CTCACACGACTCACGA-3'. cDNA was synthesized using EasyScript One-Step gDNA Removal and cDNA Synthesis SuperMix (TransGen Biotech). The RT protocol was as follows: $45^{\circ} \mathrm{C}$ for $15 \mathrm{~min}, 85^{\circ} \mathrm{C}$ for $5 \mathrm{sec}$, and end at $4^{\circ} \mathrm{C}$. qPCR was performed to analyze IL-6R expression using SYBR Premix Ex Taq (Takara Bio, Inc.). The qPCR was performed with an initial denaturation step at $95^{\circ} \mathrm{C}$ for $5 \mathrm{~min} ; 39$ cycles at $95^{\circ} \mathrm{C}$ for $10 \mathrm{sec}$ and $60^{\circ} \mathrm{C}$ for $30 \mathrm{sec}$; and a final stage at $95^{\circ} \mathrm{C}$ for $15 \mathrm{sec}, 60^{\circ} \mathrm{C}$ elongation for $1 \mathrm{~min}$ and $95^{\circ} \mathrm{C}$ for $15 \mathrm{sec}$. The following primer sequences were used: IL-6R forward, 5'-CCTCTGCATTGCCATTGT TC-3' and reverse, 5'-GAGATGAGAGGAACAAGCAC-3'; BAX forward, 5'-ACCATCATGGGCTGGACATTG-3' and reverse, 5'-CTGGAGACAGGGACATCAGTCG-3'; and Bcl-2 forward, 5'-ACTTCGCCGAGATGTCCAG-3' and reverse, 5'-CCACAATCCTCCCCCAGTTCA-3'. Quantification was performed using the $2^{-\Delta \Delta \mathrm{Cq}}$ method (16), and the expression values were normalized to the expression of GAPDH as the loading control. Each sample was run in triplicate. The expression levels of the target miRNAs and a control gene, U6, were measured simultaneously. All reactions, including the no-template controls, were performed in triplicate.

Western blotting. To assess changes in cellular protein levels, MM cells and PCs from patients were harvested after $72 \mathrm{~h}$, washed with ice-cold PBS and resuspended in $100 \mu 1$ lysis buffer (99 $\mu \mathrm{l}$ RPMI and $1 \mu \mathrm{l}$ PMSF). After $2 \mathrm{~h}$ on ice, the solution was centrifuged at $12,000 \mathrm{x} \mathrm{g}$ at $4^{\circ} \mathrm{C}$ for $20 \mathrm{~min}$. The supernatants were stored at $-80^{\circ} \mathrm{C}$ until required. Proteins $(20 \mu \mathrm{g} /$ lane $)$ were subjected to electrophoresis on a $10 \%$ SDS-gel, resolved using SDS-PAGE and transferred to nitrocellulose membranes (Bio-Rad Laboratories, Inc.). 
The membranes were blocked with $5 \%$ nonfat dried milk in Tris-buffered saline with $0.05 \%$ Tween-20 and incubated with the following primary antibodies overnight at $4^{\circ} \mathrm{C}$ : Rabbit anti-IL-6R (1:400; cat. no. ab128008; Abcam), rabbit anti-BAX (1:1,000; cat. no. ab32503; Abcam), rabbit anti-Bcl-2 (1:1,000; cat. no. ab185002; Abcam), rabbit anti-JAK2 (1:5,000; cat. no. ab108596; Abcam), rabbit anti-p-JAK2 (1:1,000; cat. no. ab32101; Abcam), mouse anti-STAT3 (1:5,000; cat. no. ab119352; Abcam) and rabbit anti-p-STAT3 (1:1,000, ab76315, Abcam). Subsequently, the membranes were incubated with horseradish peroxidase-conjugated secondary antibodies (1:2,000; cat. nos. ab6789 and ab6721; Abcam) for $2 \mathrm{~h}$ at room temperature and visualized using enhanced chemiluminescence reagent (Amersham; Cytiva).

Measurement of serum IL-6 levels. The levels of IL-6 in serum were measured using an IL-6 ELISA kit (cat. no. ab46027; Abcam) according to the manufacturer's protocol. After color development was stopped, the absorbance was measured using a computer-connected microtiter plate reader at $450 \mathrm{~nm}$. The sensitivity for IL-6 detection was $2 \mathrm{pg} / \mathrm{ml}$.

Flow cytometry analysis. A fixative-free erythrocyte lysis (Beckman Coulter, Inc.) method was used for phenotypic characterization of most participants. Intraprep permeabilization reagent (cat. no. A07803; Beckman Coulter, Inc.) was used to assess intracellular immunoglobulin levels at room temperature. The number and viability of cells obtained were assessed using trypan blue (cat. no. T8070; Solarbio) for $10 \mathrm{~min}$ at room temperature and observed under a light microscope (Olympus Corporation; magnification, 10x10) by counting the number of unstained cells. If the viability of recovered cells was $\geq 90 \%, 2 \times 10^{6}$ cells were stained with two independent 6 -color panels $\left(1 \times 10^{6}\right.$ cells each) at room temperature for $30 \mathrm{~min}$ : One tube contained CD19-FITC, CD20-PE, CD56-ECD, CD38-PECY5.5, CD138-APC and CD45-PECy7, and the other tube contained CD19-FITC, CD117-PE, CD56-ECD, CD38-PECY5.5, CD138-APC and CD45-PECy7. The intracytoplasmic panel consisted of the markers Сук-APC, Су $\lambda$-APC750, CD19-PE, CD38-FITC and CD138-APC (all antibodies were purchased from Beckman Coulter, Inc.). A total of $1 \times 10^{6}$ cells were suspended in a final volume of $200 \mu \mathrm{l} / \mathrm{tube}$ and labeled. A minimum of $5 \times 10^{6}$ events were recorded per tube in a Navios cytometer (Beckman Coulter, Inc.) with a set forward scatter (FSC) threshold of 10,000 within a maximum of $1 \mathrm{~h}$ after the final washing step of the preparation process. Data analysis was performed using Kaluza (version no. 2.1; Beckman Coulter, Inc.). After exclusion of cell doublets and debris, PCs from the remaining BM cells were mainly selected using CD138/CD38, CD45/CD38 and side scatter/FSC bivariate dot plots. CD19 and CD56 were predicted to be applicable to $\geq 90 \%$ of patients, and the markers CD20 and CD117 were considered likely to increase the percentage to $\geq 95 \%$ of patients. Assessment of cytoplasmic $\kappa / \lambda$ expression by flow cytometry is important to demonstrate clonality (with a value $>3$ or $<0.33$ representing the monoclonal light chain) $(15,17)$. Abnormal PCs were identified as CD45 negative/weakly positive, CD19 negative, strongly CD56 positive, CD117 positive, CD20 positive and cytoplasmic $\kappa$ or $\lambda$ monoclonal positive.
IL-6R expression in PCs from patients and normal controls was detected by MFC according to the manufacturer's protocol (cat. no. ab27321; Abcam). To analyze the proliferative capacity, Ki-67 expression was detected in U266 cells using MFC according to the manufacturer's protocol (cat. no. B27259; BioLegend, Inc.).

U266 cells were cultured in 60-mm petri dishes for $48 \mathrm{~h}$ at $37^{\circ} \mathrm{C}$ with $5 \% \mathrm{CO}_{2}$ and transfected with mimics or inhibitors for another $48 \mathrm{~h}$. The pro-apoptotic effects of miR-451a on U266 cells were examined by MFC following double-staining with Annexin V/FITC and propidium iodide using an Annexin V Apoptosis Detection kit (eBioscience; Thermo Fisher Scientific, Inc.) according to the manufacturer's protocol.

Immunohistochemistry. Paraffin-embedded sections of patients' BM biopsies in the department of pathology were deparaffinized using xylene and ethanol. Then, the sections were blocked by $2 \%$ bovine serum protein (Sigma-Aldrich) for $30 \mathrm{~min}$ at room temperature. Immunohistochemistry was performed on BM sections using the labeled Streptavidin-Horseradish Peroxidase (HRP) Conjugate (Invitrogen, SA10001). Antigen retrieval techniques were applied as needed for each specific antibody using IHC Antigen Retrieval Solution (Invitrogen, 00-4956-58). The following antibodies were used: IL-6R (1:4,000; cat. no. ab128008; Abcam) and CD138 (1:8,000; cat. no. ab128936; Abcam) at room temperature for $1 \mathrm{~h}$. Following the primary incubation, tissue sections were incubated with a goat anti-rabbit IgG (H\&L) secondary antibody (1:500; ab97051; Abcam) at room temperature for $1 \mathrm{~h}$. DAB was used as a substrate, and the positive signal was dark brown in color (Invitrogen, TA-060-QHDX). Samples were observed under a light microscope (Olympus Corporation; magnifications, 20x10, 10x10).

Cell viability assay. A Cell Counting Kit-8 assay was used to determine cell viability (Dojindo Molecular Technologies, Inc.) according to the manufacturer's protocol. Cell proliferation was assessed using BrdU staining (Roche Diagnostics, $\mathrm{GmbH}$ ) at room temperature for approximately $2.5 \mathrm{~h}$. The absorbance was assessed using a microplate reader (BioTek Instruments, Inc.) at a wavelength of $370 \mathrm{~nm}$.

Luciferase reporter assay. The online software TargetScan (TargetScan Human 7.0, http://www.targetscan.org/vert_70) was used to determine the association between miR-451a and IL-6R. A human IL-6R 3'-UTR with a mutation in the miR-451a seed sequence was amplified and inserted into the firefly and Renilla luciferase reporter vector pmiR-RB-REPORT (Guangzhou RiboBio Co., Ltd.) to form a mutant (Mut) vector. Cells were transfected with the luciferase reporter vectors (30 ng). Firefly and Renilla luciferase activity levels were consecutively measured according to the manufacturer's protocol (Promega Corporation) $24 \mathrm{~h}$ after transfection. The Renilla luciferase signal was normalized to the respective firefly luciferase signal.

Statistical analysis. Statistical analysis was performed using SPSS version 19.0 (IBM Corp.). The $\chi^{2}$ test was used to determine whether sex and age ratios significantly differed between the experimental group and the controls. Data are presented as 

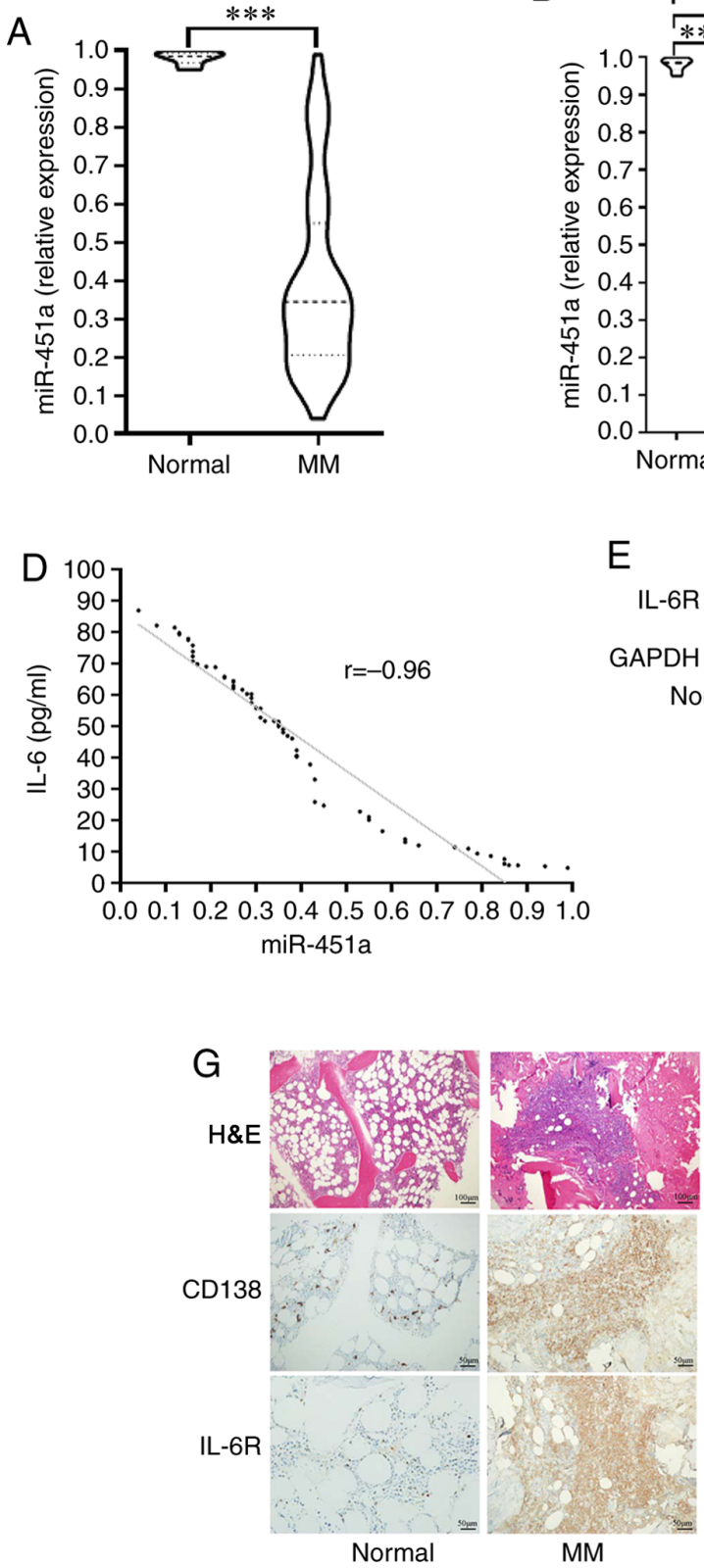

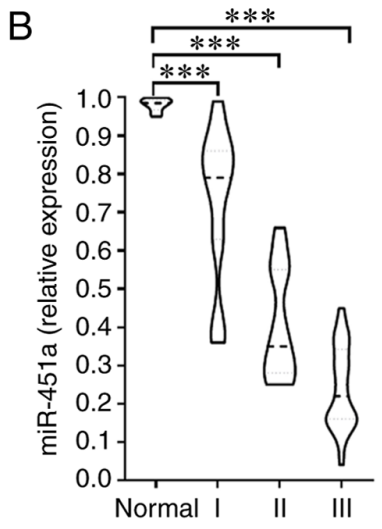

$\mathrm{E}$
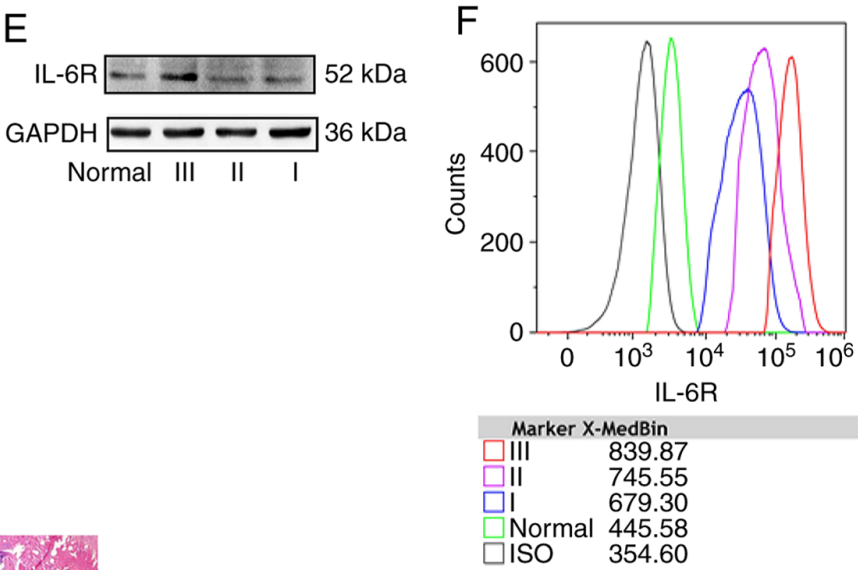

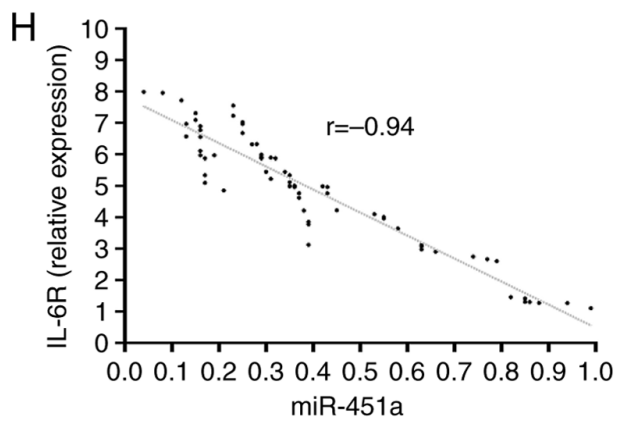

Figure 1. miR-451a expression is downregulated in patients with MM. (A) Relative expression levels of miR-451a in normal controls and patients with MM. (B) Relative expression levels of miR-451a in patients with MM based on R-ISS stage. (C) Relative expression levels of IL-6 in normal controls and patients with MM. (D) Correlation between miR-451a and IL-6 levels. (E) Western blot showing the levels of IL-6R expression in plasma cells from bone marrow. (F) Intensity of IL-6R expression as determined by flow cytometry. (G) Immunohistochemical analysis of tumor cells. Tumor cells were positive for CD138 and IL-6R. Bar: $100 \mu \mathrm{m}$ in H\&E. Bar:50 $\mu \mathrm{m}$ in immunohistochemical analysis. (H) Correlation between miR-451a and IL-6R levels. ${ }^{* * *}$ P<0.0001. miR, microRNA; MM, multiple myeloma; IL-6, interleukin-6; IL-6R, IL-6 receptor; H\&E, hematoxylin and eosin.

the means \pm standard deviations of three independent repeats. A one-way ANOVA with post hoc Tukey's test was used to compare differences between multiple groups. Correlation coefficients were calculated using Spearman's correlation test to analyze correlations between two variables. The $\chi^{2}$ test was used to compare frequencies between two groups. A two-sided $\mathrm{P}<0.05$ was considered indicative of a statistically significant difference.

\section{Results}

miR-451a expression in the BM of patients with MM at diagnosis. Among the patients, 15 (22.7\%) had R-ISS stage I
MM, 17 (25.8\%) had R-ISS stage II MM, and 34 (51.5\%) had R-ISS stage III MM. These patients received immunomodulatory drugs or PIs for induction therapy without autologous stem cell transplantation. miR-451a expression was low in MM patients at $0.39 \pm 0.13$-times that in normal subjects $(\mathrm{t}=7.66, \mathrm{P}<0.0001)$ (Fig. 1A). Among the 66 patients with MM, the median level of miR-451a in patients with R-ISS stage I MM was $0.72 \pm 0.05$-times that in normal subjects. The median level in patients with R-ISS stage II MM was $0.40 \pm 0.03$-times that in normal subjects. R-ISS stage III patients showed the lowest level $(0.24 \pm 0.01$-times that in normal subjects; $\mathrm{F}=101.14, \mathrm{P}<0.0001$; Fig. $1 \mathrm{~B})$. The concentration of IL-6 in MM patients was significantly higher than 

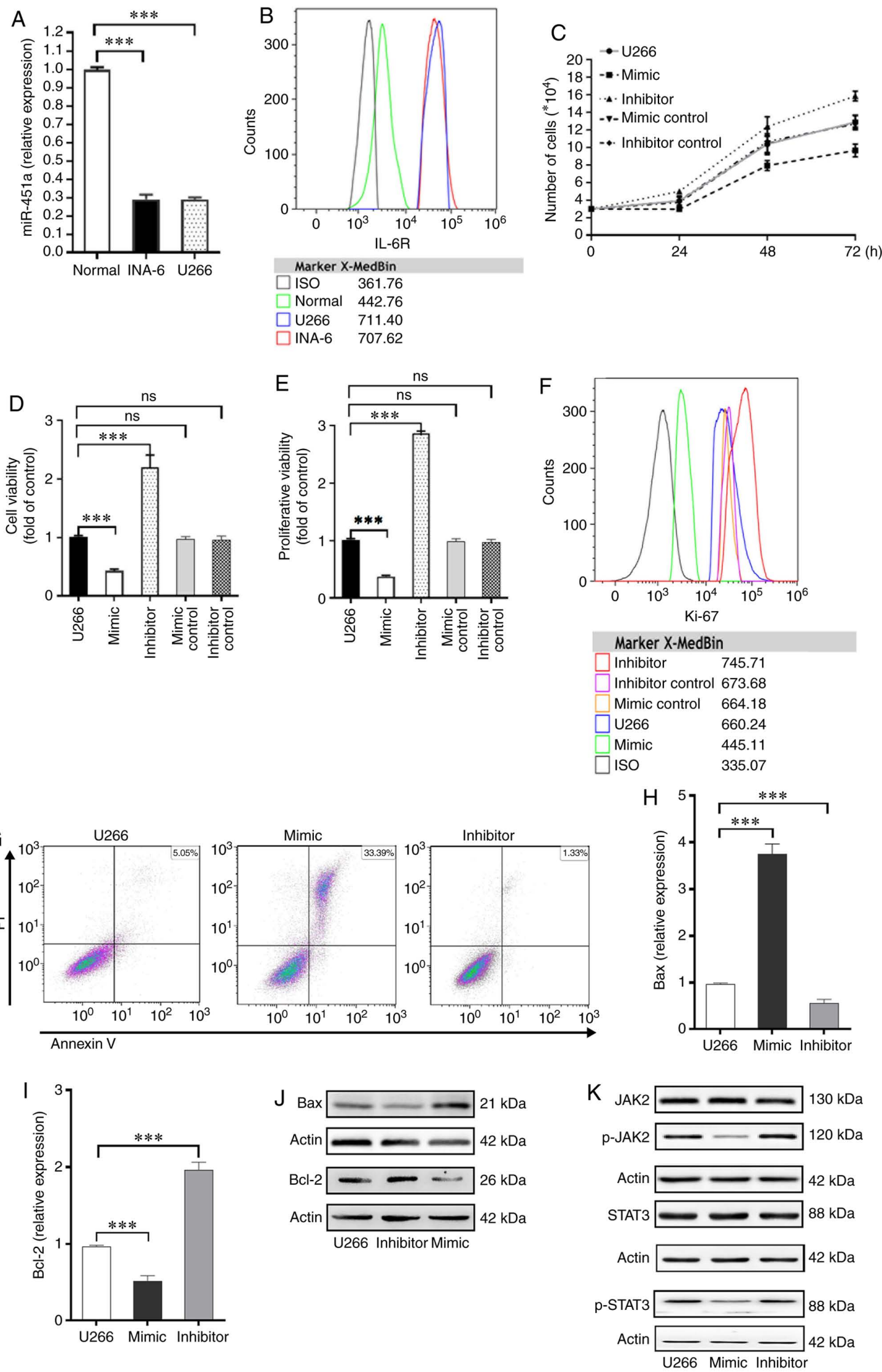

Figure 2. miR-451a mimic inhibits proliferation and induces apoptosis in MM cells. (A) Relative expression levels of miR-451a in MM cells. (B) IL-6R expression was analyzed by MFC. (C) Growth curves of cell lines transfected with the miR-451a mimic or inhibitor. (D) Cell Counting Kit-8 assay for cell viability. (E) Proliferative capacity of transfected cells assessed using a BrdU assay. (F) Ki-67 expression analysis as determined using MFC. (G) Apoptosis in U266 cells transfected with the miR-451a mimic or inhibitor. (H and I) mRNA expression levels of Bax and Bcl-2 in the transfected U266 cells. (J) Western blot analyses of Bax and Bcl-2 in the transfected U266 cells. (K) Western blot showing JAK2 and STAT3 inactivation in cells treated with the mimic. ${ }^{* * *} \mathrm{P}<0.0001$. miR, microRNA; MM, multiple myeloma; IL-6, interleukin-6; IL-6R, IL-6 receptor; MFC, multiparameter flow cytometry; ns, not significant; p-, phospho-; ISO, isotype. 

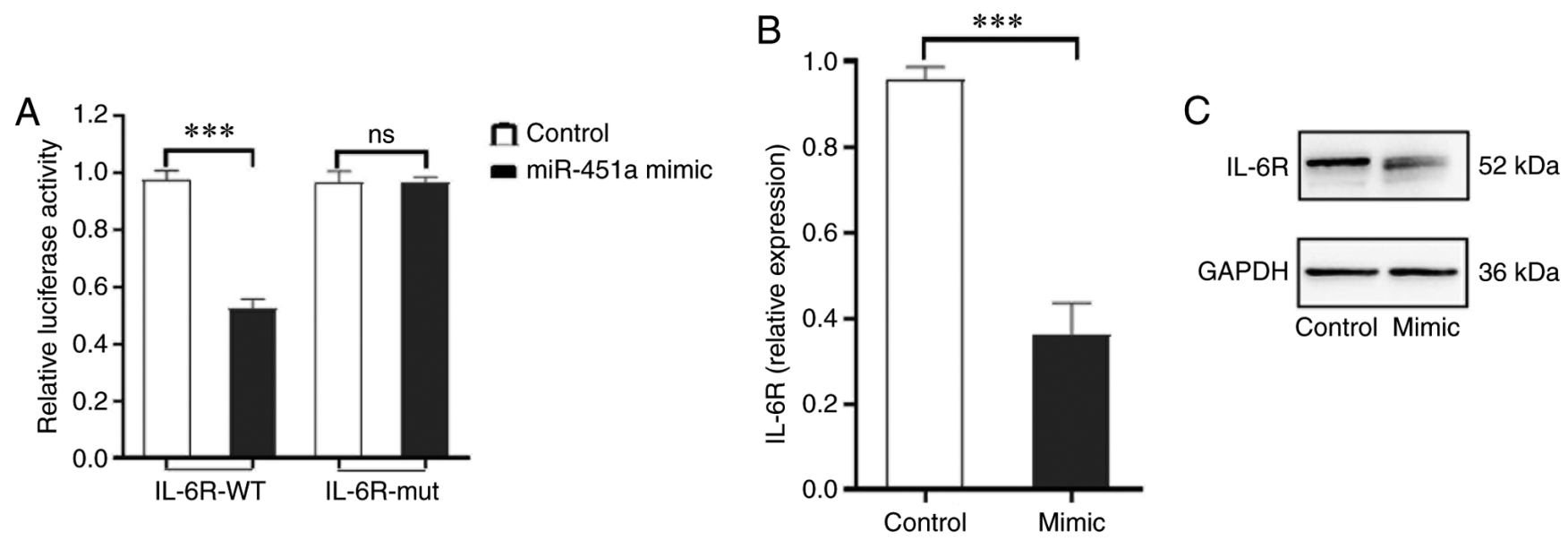

Figure 3. IL-6R is the direct target of miR-451a. (A) Binding between IL-6R and miR-451a was assessed using a dual-luciferase reporter assay. (B) mRNA and $(\mathrm{C})$ protein expression levels of IL-6R in miR-451a mimic-transfected cells. ${ }^{* * *} \mathrm{P}<0.0001$. miR, microRNA; IL-6R, interleukin-6 receptor; mut, mutant; WT, wild-type.

that in the normal controls $(\mathrm{t}=5.62, \mathrm{P}<0.0001$; Fig. $1 \mathrm{C})$ and was inversely related to the levels of miR-451a $(r=-0.96$, $\mathrm{P}<0.0001$; Fig. 1D).

Compared with those from the controls, PCs from MM patients, particularly R-ISS stage III patients, showed upregulated expression of IL-6R as indicated by western blot analysis (Fig. 1E). Furthermore, the MFC results showed differences in the expression intensity of IL-6R among the different R-ISS stages, with stage III patients exhibiting the highest intensity (Fig. 1F). BM histopathology showed that the tumor cells formed foci and were strongly positive for both CD138 (plasma positive) and IL-6R (membrane positive) (Fig. 1G). IL-6R expression was negatively correlated with the level of miR-451a (r=-0.94, P<0.0001; Fig. 1H).

The mechanism underlying miR-45la regulation of myeloma cell proliferation and apoptosis. The levels of miR-451a among INA-6 cells, U266 cells and PCs from normal controls were compared. The expression of miR-451a was $0.28 \pm 0.02$-times normal levels in INA-6 cells and 0.29 \pm 0.03 -times normal levels in U266 cells ( $\mathrm{F}=478.13, \mathrm{P}<0.0001$; Fig. 2A). The expression of IL-6R was analyzed by MFC. IL-6R expression on the surfaces of U266 and INA-6 cells was significantly higher than on the surfaces of normal cells. The median fluorescence intensity (MFI) of U266 cells was 711.4, the MFI of INA-6 cells was 707.62, and the MFI of normal cells was only 442.76 (Fig. 2B).

In U266 cells transfected with the miR-451a mimic or miR-451a inhibitor, miR-451a expression was increased or reduced, respectively. Furthermore, the levels of miR-451a in cells transfected with the mimic control and inhibitor control did not differ from those in the untransfected U266 cells (Fig. S1). Among the groups, U266 cells transfected with the miR-451a inhibitor exhibited the highest proliferation rate after $72 \mathrm{~h}$, whereas the miR-451a mimic-transfected cells exhibited the lowest levels of proliferation. Cells treated with the mimic control and inhibitor control did not significantly differ from the untransfected U266 cells with regard to proliferation (Fig. 2C). Treatment with the miR-451 mimic decreased the viability of U266 cells by $57.3 \% \pm 3.1 \%$ and decreased the proliferative ability of U266 cells by $62.6 \% \pm 2.6 \%$. The miR-451a inhibitor showed the opposite effects $(\mathrm{F}=54.628, \mathrm{P}<0.0001$, and $\mathrm{F}=2749.244$, $\mathrm{P}<0.0001$, respectively). The groups treated with the mimic control and inhibitor control did not significantly differ from the untransfected U266 cells (P>0.05; Fig. 2D and E). Using MFC, the miR-451a mimic was found to significantly reduce the intensity of Ki-67 expression in U266 cells, whereas transfection with the miR-451a inhibitor significantly increased Ki-67 expression. The mimic control and inhibitor control did not affect the intensity of Ki-67 expression in U266 cells compared with the untransfected U266 cells (Fig. 2F).

The miR-451a mimic also significantly increased the rate of apoptosis in U266 cells from $5.05 \%$ (control) to $33.39 \%$ (transfected U266 cells) (Fig. 2G). The expression of the apoptotic protein Bax was significantly upregulated in the mimic-transfected cells, and the expression of the antiapoptotic protein, Bcl-2, was significantly decreased. When miR-451a was inhibited, the opposite trend was observed in the mRNA (Bcl-2, $\mathrm{F}=172.577, \mathrm{P}<0.0001$; $\mathrm{Bax}$, $\mathrm{F}=112.922, \mathrm{P}<0.0001$; Fig. $2 \mathrm{H}$ and $\mathrm{I})$ and protein expression levels of Bcl-2 and Bax (Fig. 2J). The JAK2/STAT3 pathway is the classical pathway stimulated by IL-6, and its cancer-promoting effects are well established $(18,19)$. Thus, the expression levels of JAK 2 and STAT 3 and their phosphorylated forms were assessed. Total JAK2 and STAT3 levels among the three groups did not differ. However, the levels of phospho-(p-)JAK2 and p-STAT3 were significantly lower in U266 cells treated with the miR-451a mimic than in the control cells (Fig. 2K).

miR-451a target verification and assessment of the target gene IL-6R. 3'-UTR luciferase reporter assays were used to assess the binding of $\mathrm{miR}-451 \mathrm{a}$ to its targets. Luciferase activity in cells containing the wild-type (WT) vector was reduced in cells transfected with miR-451a and the 3'UTR of IL-6R by $0.36 \pm 0.07$-fold. Cells transfected with a miR-451a Mutant (Mut) vector did not exhibit a decrease in luciferase activity (Fig. 3A). Furthermore, the miR-451a mimic control had no effect on the luciferase activity of the WT vector or Mut vector. These results showed that miR-451a directly 
repressed IL-6R by binding to the 3'-UTR. Additionally, in cells transfected with the miR-451a mimic, IL-6R mRNA (Fig. 3B) and protein expression levels (Fig. 3C) were significantly decreased.

\section{Discussion}

IL-6 plays a crucial role in the pathogenesis of MM, and the discovery of the involvement of IL-6 in MM is of notable significance (20). IL-6 is critical for the growth and survival of malignant PCs, and several factors and mechanisms can increase IL-6 levels (21). However, IL-6 must bind to its receptor to elicit its effects as IL-6R transduces the IL-6 signal (5). The molecular mechanisms regulating IL-6R expression have not yet been fully elucidated (22). In the present study, miR-451a was shown to target IL-6R to offset the effects induced by IL-6. To the best of our knowledge, the present study is the first to highlight miR-451a as a vital antitumor factor in MM.

Abnormal expression of miR-451a is negatively correlated with the degree of malignancy in several types of malignant tumors (23). Recently, miR-451a was shown to be significantly downregulated in patients with $\mathrm{MM}$ and was correlated with a poor clinical prognosis (24), which is consistent with the results of the present study. However, the mechanisms by which miR-451a expression is downregulated remain unclear. In addition, long non-coding (lnc)RNAs have been shown to sponge miRNAs, resulting in a decrease in miRNA expression, and LINC00657, AC084082.3 and LINC00657 have been found to interact with miR-451a in BM (25). However, in MM, the expression levels of the lncRNAs that sponge miR-451a are unknown. IL-6R was confirmed to be a target of miR-451a in the present study, indicating that miR-451a was associated with the IL-6R/JAK2/STAT3 pathway. Whether lncRNAs that sponge miR-451a are also associated with this pathway, which exhibits a high level of activity in MM, will be addressed in future studies.

The downstream mechanism by which downregulation of miR-451a influences MM is unknown. In osteosarcoma, miR-451a inhibits the proliferation, migration and angiogenesis of cancer cells by silencing IL-6R (26). In the present study, miR-451a levels were negatively correlated with the R-ISS stage and IL-6 and IL-6R levels. The dual-luciferase reporter assays confirmed the relationship between miR-451a and IL-6R. Therefore, miR-451a may function through the IL-6R/JAK2/STAT3 pathway. Further analyses verified that miR-451a altered the levels of pJAK2 and pSTAT3 (active forms) to induce apoptosis of myeloma cells with no changes in the levels of total JAK2 and STAT3; this mechanism may apply to clinical transformation. Thus, miR-451a may serve as a potential biomarker for the prediction of patient outcomes. In addition to signaling via the JAK2/STAT3 pathway, IL-6 can also promote the proliferation of myeloma cells through the Ras/MAPK pathway (27) and reduce apoptosis of MM cells by regulating the PI3K/AKT pathway (28). Furthermore, IL-6 interacts with VEGF to promote angiogenesis, migration and invasion (29). Several pathways activated by IL-6 result in activation of $\mathrm{NF}-\mathrm{KB}$ (30). Therefore, miR-451a may exert its effects through other mechanisms and signaling pathways, which may be tumor-type dependent. However, these hypotheses must be confirmed in future studies.

In conclusion, the present study showed that miR-451a specifically silences IL-6R-induced myeloma cell apoptosis and promotes JAK2/STAT3 pathway inactivation to exert its antitumor effects. Thus, miR-451a may be an attractive alternative target for the mapping of tumor loads in real time and for MM treatment via novel strategies.

\section{Acknowledgements}

The authors of the present study would like to thank Mr. Yi Shi (Sichuan Academy of Medical Sciences and Sichuan Provincial People's Hospital, School of Medicine, University of Electronic Science and Technology of China), for his ongoing advice and assistance in analyzing the data. The authors would also like to thank Dr Gramatzki (Laboratory of Hematology, University of Liège, Belgium), for kindly providing the cell line used in the present study.

\section{Funding}

The present study was supported by the Health Commission of Sichuan Province (grant no. 150193), the Commission of the Cardre Health Care in Sichuan Province (grant no. 2017-228), the Science \& Technology Department of Sichuan Province (grant no. 19YJ0593), the Sichuan Provincial People's Hospital (grant no. 2018LY03), the Chengdu Science and Technology Bureau (grant no. 2015-HM01-00470-SF) and the Science \& Technology Department of Sichuan Province (grant no. 2020YFS0433).

\section{Availability of data and materials}

The datasets used and/or analyzed during the present study are available from the corresponding author upon reasonable request.

\section{Authors' contributions}

LZ, ZYX, XJ, YH, JBZ, TJ and JC participated in the design and interpretation of the studies, the analysis of the data and in editing the manuscript. LZ, ZYX and XJ performed the experiments. YH and JBZ performed the statistical analysis and the follow-up of the patients. LZ, TJ, and JC wrote the manuscript. All authors read and approved the final manuscript.

\section{Ethics approval and consent to participate}

The present study was approved by the Institutional Review Board of Sichuan Provincial Peoples' Hospital of China (Chengdu, Sichuan; approval no. 2018-49). Written informed consent was obtained from each eligible patient, and the entire study was performed in accordance with the guidelines stated in the Declaration of Helsinki.

\section{Patient consent for publication}

Not applicable. 


\section{Competing interests}

The authors declare that they have no competing interests.

\section{References}

1. Chang SH, Luo S, Thomas TS, O'Brian KK, Colditz GA, Carlsson NP and Carson KR: Obesity and the transformation of monoclonal gammopathy of undetermined significance to multiple myeloma: A population-based cohort study. J Natl Cancer Inst 109: djw264, 2016.

2. Popovic M, Lao N, Bedard G, Zeng L, Zhang L, Cella D, Beaumont JL, Chiu N, Chiu L, Lam H, et al: Quality of life in patients with advanced cancer using the functional assessment of cancer therapy-general assessment tool: A literature review. World J Oncol 4: 8-17, 2013.

3. Tandon N, Rajkumar SV, LaPlant B, Pettinger A, Lacy MQ Dispenzieri A, Buadi FK, Gertz MA, Hayman SR, Leung N, et al: Clinical utility of the revised international staging system in unselected patients with newly diagnosed and relapsed multiple myeloma. Blood Cancer J 7: e528, 2017.

4. Furukawa M, Ohkawara H, Ogawa K, Ikeda K, Ueda K, Shichishima-Nakamura A, Ito E, Imai JI, Yanagisawa Y, Honma R, et al: Autocrine and paracrine interactions between multiple myeloma cells and bone marrow stromal cells by growth arrest-specific gene 6 cross-talk with interleukin-6. J Biol Chem 292: 4280-4292, 2017.

5. Mishra AK and Dingli D: Metformin inhibits IL-6 signaling by decreasing IL-6R expression on multiple myeloma cells. Leukemia 33: 2695-2709, 2019.

6. De Oliveira MB, Fook-Alves VL, Eugenio AIP, Fernando RC, Sanson LFG, de Carvalho MF, Braga WMT, Davies FE and Colleoni GWB: Anti-myeloma effects of ruxolitinib combined with bortezomib and lenalidomide: A rationale for JAK/STAT pathway inhibition in myeloma patients. Cancer Lett 403: 206-215, 2017

7. Altuvia Y, Landgraf P, Lithwick G, Elefant N, Pfeffer S, Aravin A, Brownstein MJ, Tuschl T and Margalit H: Clustering and conservation patterns of human microRNAs. Nucleic Acids Res 33: 2697-2706, 2005.

8. Wang R, Wang ZX, Yang JS, Pan X, De W and Chen LB: MicroRNA-451 functions as a tumor suppressor in human non-small cell lung cancer by targeting ras-related protein 14 (RAB14). Oncogene 30: 2644-2658, 2011.

9. Huang JY, Zhang K, Chen DQ, Chen J, Feng B, Song H, Chen Y, Zhu Z, Lu L, De W, et al: MicroRNA-451: Epithelial-mesenchymal transition inhibitor and prognostic biomarker of hepatocellular carcinoma. Oncotarget 6 : 18613-18630, 2015.

10. Kim Y, Powathil G, Kang H, Trucu D, Kim H, Lawler S and Chaplain M: Strategies of eradicating glioma cells: A multi-scale mathematical model with MiR-451-AMPK-mTOR control. PLoS One 10: e114370, 2015.

11. Yuan J, Lang J, Liu C, Zhou K, Chen L and Liu Y: The expression and function of miRNA-451 in osteosarcoma. Med Oncol 32: 324, 2015.

12. Gits CM, van Kuijk PF, Jonkers MB, Boersma AWM, Smid M, van Ijcken WF, Coindre JM, Chibon F, Verhoef C, Mathijssen RHJ, et al: MicroRNA expression profiles distinguish liposarcoma subtypes and implicate miR-145 and miR-451 as tumor suppressors. Int J Cancer 135: 348-361, 2014.

13. Liu X, Zhang A, Xiang J, Lv Y and Zhang X: miR-451 acts as a suppressor of angiogenesis in hepatocellular carcinoma by targeting the IL-6R-STAT3 pathway. Oncol Rep 36: 1385-1392, 2016.

14. Chng WJ, Dispenzieri A, Chim CS, Fonseca R, Goldschmidt H, Lentzsch S, Munshi N, Palumbo A, Miguel JS, Sonneveld P, et al: IMWG consensus on risk stratification in multiple myeloma. Leukemia 28: 269-277, 2014

15. Kumar S, Paiva B, Anderson KC, Durie B, Landgren O, Moreau P, Munshi N, Lonial S, Bladé J, Mateos MV, et al: International myeloma working group consensus criteria for response and minimal residual disease assessment in multiple myeloma. Lancet Oncol 17: e328-e346, 2016.
16. Livak KJ and Schmittgen TD: Analysis of relative gene expression data using real-time quantitative PCR and the 2(-Delta Delta C(T)) method. Methods 25: 402-408, 2001

17. Flores-Montero J, Sanoja-Flores L, Paiva B, Puig N, García-Sánchez O, Böttcher $S$, van der Velden VHJ, Pérez-Morán JJ, Vidriales MB, García-Sanz R, et al: Next generation flow for highly sensitive and standardized detection of minimal residual disease in multiple myeloma. Leukemia 31: 2094-2103, 2017.

18. Kolosenko I, Grander D and Tamm KP: IL-6 activated JAK/STAT3 pathway and sensitivity to Hsp90 inhibitors in multiple myeloma. Curr Med Chem 21: 3042-3047, 2014.

19. Liu X, Wang J, Wang H, Yin G, Liu Y, Lei X and Xiang M: REG3A accelerates pancreatic cancer cell growth under IL-6-associated inflammatory condition: Involvement of a REG3A-JAK2/STAT3 positive feedback loop. Cancer Lett 362: 45-60, 2015.

20. Suematsu S, Hibi M, Sugita T, Saito M, Murakami M, Matsusaka T, Matsuda T, Hirano T, Taga T and Kishimoto T: Interleukin 6 (IL-6) and its receptor (IL-6R) in myeloma/plasmacytoma. Curr Top Microbiol Immunol 166: 13-22, 1990.

21. Piddock RE, Marlein CR, Abdul-Aziz A, Shafat MS, Auger MJ, Bowles KM and Rushworth SA: Myeloma-derived macrophage inhibitory factor regulates bone marrow stromal cell-derived IL-6 via c-MYC. J Hematol Oncol 11: 66-69, 2018.

22. Birmann BM, Neuhouser ML, Rosner B, Albanes D, Buring JE, Giles GG, Lan Q, Lee IM, Purdue MP, Rothman N, et al: Prediagnosis biomarkers of insulin-like growth factor-1, insulin, and interleukin-6 dysregulation and multiple myeloma risk in the multiple myeloma cohort consortium. Blood 120: 4929-4937, 2012.

23. Zhao S, Li J, Zhang G, Wang Q, Wu C, Zhang Q, Wang H, Sun P, Xiang $\mathrm{R}$ and Yang S: Exosomal miR-451a functions as a tumor suppressor in hepatocellular carcinoma by targeting LPIN1. Cell Physiol Biochem 53: 19-35, 2019.

24. Meng YB, He X, Huang YF, Wu QN, Zhou YC and Hao DJ: Long noncoding RNA CRNDE promotes multiple myeloma cell growth by suppressing miR-451. Oncol Res 25: 1207-1214, 2017.

25. Balakrishnan I, Yang X, Brown J,Ramakrishnan A, Torok-Storb B, Kabos P, Hesselberth JR and Pillai MM: Genome-wide analysis of miRNA-mRNA interactions in marrow stromal cells. Stem Cells 32: 662-673, 2014

26. Liu SY, Deng SY, He YB and Ni GX: miR-451 inhibits cell growth, migration and angiogenesis in human osteosarcoma via down-regulating IL-6R. Biochem Biophys Res Commun 482: 987-993, 2017.

27. Gocke CB, McMillan R, Wang Q, Begum A, Penchev VR, Ali SA, Borrello I, Huff CA and Matsui W: IQGAP1 scaffold-MAP kinase interactions enhance multiple myeloma clonogenic growth and self-renewal. Mol Cancer Ther 15: 2733-2739, 2016

28. Mimura N, Hideshima T, Shimomura T, Suzuki R, Ohguchi H, Rizq O, Kikuchi S, Yoshida Y, Cottini F, Jakubikova J, et al: Selective and potent Akt inhibition triggers anti-myeloma activities and enhances fatal endoplasmic reticulum stress induced by proteasome inhibition. Cancer Res 74: 4458-4469, 2014.

29. Berenstein R, Nogai A, Waechter M, Blau O, Kuehnel A, Schmidt-Hieber M, Kunitz A, Pezzutto A, Dörken B and Blau IW: Multiple myeloma cells modify VEGF/IL-6 levels and osteogenic potential of bone marrow stromal cells via Notch/miR-223. Mol Carcinog 55: 1927-1939, 2016.

30. Matthews GM, de Matos Simoes R, Dhimolea E, Sheffer M, Gandolfi S, Dashevsky O, Sorrell JD and Mitsiades CS: NF- $\kappa$ B dysregulation in multiple myeloma. Semin Cancer Biol 39: 68-76, 2016.

This work is licensed under a Creative Commons Attribution-NonCommercial-NoDerivatives 4.0 International (CC BY-NC-ND 4.0) License. 